\title{
Comportamentos de risco para doenças sexualmente transmissíveis em caminhoneiros no Brasil
}

\author{
Sheila Araujo Teles, ${ }^{1}$ Marcos André de Matos, ${ }^{2}$ Karlla Antonieta Amorim Caetano, ${ }^{1}$ \\ Ludmila Araujo Costa, ${ }^{2}$ Divânia Dias da Silva França, ${ }^{2}$ Grécia Carolina Pessoni, ${ }^{2}$ \\ Sandra Maria Brunini ${ }^{2}$ e Regina Maria Bringel Martins ${ }^{3}$
}

Como citar

Teles AS, Matos MA, Caetano KAA, Costa LA, França DDS, Pessoni GC, et al. Comportamentos de risco para doenças sexualmente transmissíveis em caminhoneiros no Brasil. Rev Panam Salud Publica. 2008;24(1):25-30.

RESUMO Objetivo. Avaliar a freqüência de doenças sexualmente transmissíveis (DST) e os fatores de risco a elas associados conforme auto-relato em caminhoneiros de rota longa no Brasil.

Método. De outubro de 2005 a outubro de 2006, foram entrevistados 641 caminhoneiros de rota longa que circulam na BR-153, uma rodovia federal que atravessa o Brasil de sul a norte. $U m$ roteiro estruturado foi usado para a coleta de dados sociodemográficos. Os caminhoneiros responderam ainda a um questionário auto-aplicável sobre comportamentos de risco e antecedentes de DST. Os dados foram ajustados e analisados por regressão logística.

Resultados. Dos 641 entrevistados, $620(96,7 \%)$ responderam sobre antecedentes de DST. Desses, 35,6\% (IC95\%: 31,9 a 39,6) referiram história presente ou passada de DST. A idade superior a 30 anos foi estatisticamente associada ao relato de DST. Ainda, os caminhoneiros que relataram o uso de anfetaminas ("rebite") (razão de chances, OR: 1,7; IC95\%: 1,1 a 2,6), antecedentes prisionais (OR: 2,2; IC95\%: 1,2 a 4,2) e relacionamento sexual com profissionais do sexo (OR: 1,9; IC95\%: 1,3 a 2,8) apresentaram maior chance de relato de DST.

Conclusão. Os resultados deste estudo ratificam a elevada vulnerabilidade dos caminhoneiros brasileiros às DST e evidenciam a importância de programas específicos de prevenção de doenças e promoção da saúde para esse grupo-alvo, que vive em constante deslocamento, sendo eficaz em disseminar as DST.

Palavras-chave Caminhoneiros, doenças sexualmente transmissíveis, fatores de risco, Brasil.

As doenças sexualmente transmissíveis (DST) são importantes causas de

\footnotetext{
1 Universidade Federal de Goiás, Faculdade de Enfermagem. Enviar correspondência para Sheila Araújo Teles no seguinte endereço: Rua 227, quadra 68, s/n, CEP 74605-080, Goiânia, GO, Brasil. Fone: +55-62-3521.1822; fax +55-62-3521.1807; e-mail: sheila@ fen.ufg.br, sheilaa@terra.com.br

2 Universidade Federal de Goiás, Goiânia (GO), Brasil.

3 Universidade Federal de Goiás, Instituto de Patologia Tropical e Saúde Pública, Goiânia (GO), Brasil.
}

doença aguda, infertilidade, incapacidade e morte. Estima-se que ocorram, anualmente, cerca de 340 milhões de casos novos de DST em todo o mundo, sendo de 10 a 12 milhões no Brasil (1). Essas infecções ocorrem, principalmente, em adultos jovens de países em desenvolvimento, sendo normalmente associadas a fatores de ordem sociocultural $(2,3)$. Ainda, a presença de uma DST pode facilitar a aquisição e a transmissão da infecção pelo vírus da imunodeficiência humana (HIV) (1).

Os caminhoneiros são uma população predominantemente masculina. Muitos permanecem longos períodos fora de casa, longe dos familiares, da esposa ou companheira, e apresentam comportamentos de risco para as DST, como uso de bebidas alcoólicas e outras substâncias psicoativas, relações sexuais sem proteção, múltiplos par- 
ceiros e relações sexuais com profissionais do sexo (4-8). Diversos estudos têm mostrado índices elevados de DST nessa população $(4,5,9,10)$. No Brasil, uma investigação realizada junto a 300 motoristas de caminhão que aguardavam carregamento no Porto de Santos, no Estado de São Paulo, encontrou relato de DST em $47 \%$ dos indivíduos (11). Ainda, 12,7\% apresentavam positividade global para a sífilis (FTA-Ac e VDRL), e 1,3\% para o anti-HIV.

Para a elaboração de políticas públicas de promoção da saúde e prevenção de agravos, é imperativo conhecer a população-alvo e identificar as suas necessidades e vulnerabilidades. $\mathrm{O}$ relato de sintomas de DST parece ser uma estratégia sensível e de baixo custo para a identificação de grupos em elevado risco para essas doenças (3). Os caminhoneiros de rota longa vivem em constante deslocamento geográfico e possuem um estilo de vida próprio, que parece facilitador da disseminação de DST. Contudo, ainda são poucas as informações sobre as DST nesse grupo populacional em nosso País.

Assim, o objetivo do presente estudo foi investigar a freqüência de DST e os fatores de risco a elas associados conforme auto-relato em caminhoneiros que trafegam em uma grande rodovia federal que interliga as regiões Sul e Norte do Brasil.

\section{MATERIAIS E MÉTODOS}

O presente estudo transversal foi realizado em Goiânia, Estado de Goiás, no período de outubro de 2005 a outubro de 2006. A população incluiu caminhoneiros de rota longa que circulam na BR-153, uma importante rodovia federal que liga as regiões Sul (Aceguá, Estado do Rio Grande do Sul) e Norte (Marabá, Estado do Pará) do Brasil, com $4355 \mathrm{~km}$ de extensão (figura 1). São caminhoneiros de rota longa aqueles que fazem regularmente percursos de no mínimo $1000 \mathrm{~km}$.

A coleta de dados foi realizada uma vez por mês, em fins de semana (aos sábados ou domingos), no horário matutino ou vespertino, em um grande posto de combustível situado às mar-

FIGURA 1. Apresentação esquemática da rodovia federal BR-153, Brasil, 2006

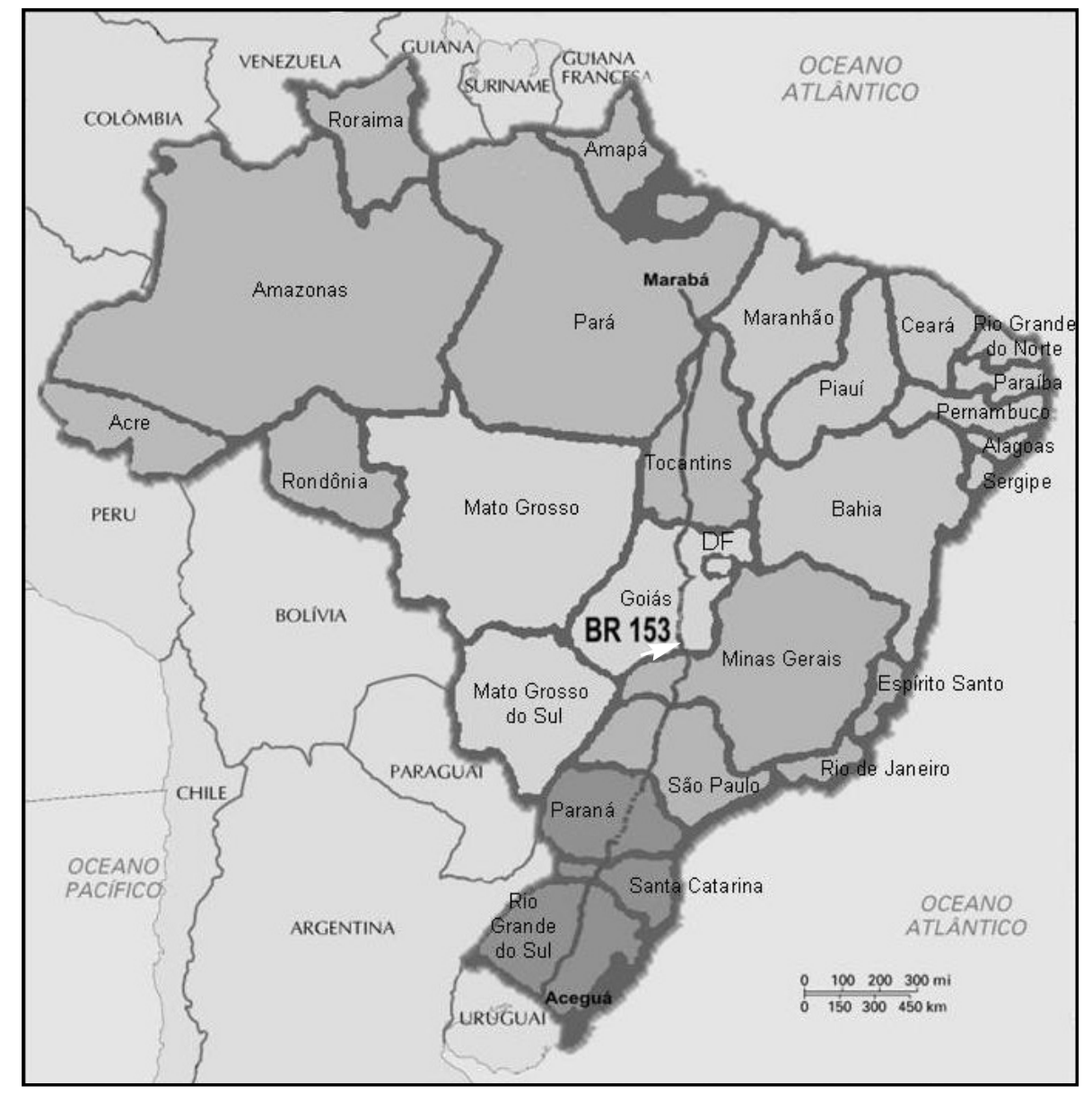

gens da BR-153, no km 1 296. Esse estabelecimento é considerado como ponto estratégico para o descanso de motoristas e o reabastecimento dos veículos de carga, atendendo cerca de mil caminhoneiros por dia em finais de semana e feriados.

Foram considerados elegíveis para o estudo os caminhoneiros que possuíam no mínimo 12 meses de profissão e que realizavam rotineiramente viagens com distância mínima de 1000 $\mathrm{km}$. Os participantes do estudo foram recrutados sucessivamente, na ordem de chegada ao posto para reabastecimento de seus veículos. Antes do início da entrevista, os indivíduos recebiam informações sobre os objetivos do estudo, sendo garantido o sigilo das informações obtidas. Caso aceitassem, assinavam, após leitura e esclarecimen- tos de dúvidas, o termo de consentimento livre e esclarecido. A seguir, eram entrevistados para a obtenção de dados sociodemográficos e ocupacionais, com base em um roteiro estruturado, e respondiam um questionário auto-aplicável, contendo perguntas sobre antecedentes de DST e comportamentos de risco. Quatro auxiliares de pesquisa foram previamente treinados para a realização das entrevistas e para o esclarecimento de dúvidas em relação ao questionário auto-aplicável. Para identificar possíveis vieses de seleção, foram obtidos também os dados sociodemográficos (sexo, idade, escolaridade e estado civil) dos caminhoneiros que se recusaram a participar do estudo.

Definiu-se como variável de desfecho o relato de história de DST (em 
algum momento da vida). A ocorrência de DST foi definida como presença de corrimento uretral ou úlcera genital de origem não traumática, conforme abordagem sindrômica de DST (12), ou diagnóstico médico-laboratorial auto-relatado. Foram consideradas variáveis de predição: dados sociodemográficos (sexo, idade, escolaridade, naturalidade e renda familiar); dados ocupacionais (anos de atividade como caminhoneiro e duração média das viagens por mês); e comportamentos de risco, inclusive intensidade do uso de preservativos com parceira fixa (esposa/companheira) e ocasional (profissionais do sexo), história presente ou passada de relações sexuais com profissionais do sexo, relações sexuais sob efeito de drogas psicoativas, uso de drogas ilícitas, uso de "rebite" (anfetamina) e prisão.

Os dados obtidos foram analisados no programa estatístico Statistical Package for the Social Sciences (SPSS), versão 13.0. Os índices de prevalência foram calculados com intervalo de confiança de 95\% (IC95\%). Inicialmente, foi realizada a análise univariada, estimando-se a associação entre a chance de relato de DST e as variáveis investigadas. A seguir, as variáveis que apresentaram significância estatística foram incluídas em um modelo de regressão logística hierárquica (13). O teste do qui-quadrado $\left(\chi^{2}\right)$ e o teste exato de Fisher foram utilizados para testar a significância das diferenças entre proporções. Valores de $P<0,05$ foram considerados como estatisticamente significativos.

Este estudo foi aprovado pelo Comitê de Ética em Pesquisa Médica Humana e Animal do Hospital Materno Infantil da Secretaria Estadual de Saúde de Goiás.

\section{RESULTADOS}

No período do estudo, 771 caminhoneiros foram elegíveis, dos quais 641 $(83,1 \%)$ concordaram em participar da investigação. Os dados sociodemográficos dos caminhoneiros que não aceitaram participar do estudo foram comparados aos daqueles que aceitaram, não sendo observada diferença estatística entre os dois grupos $(P>0,05)$.

As características sociodemográficas dos indivíduos que participaram do estudo são apresentadas na tabela 1. Praticamente a totalidade dos caminhoneiros era do sexo masculino $(99,2 \%)$. A maioria era casada $(77,7 \%)$, natural das regiões Sul $(40,2 \%)$ e Sudeste $(41,2 \%)$ do Brasil, tinha idade entre 31 e 50 anos $(62,9 \%)$ e renda igual ou inferior a 6 salários mínimos (77,1\%). Em relação à escolaridade, quase um terço possuía no máximo 4 anos de escolaridade.

Do total de participantes, 620 (96,7\%) responderam sobre antecedentes de DST e, desses, 221 (35,6\%; IC95\%: 31,9 a 39,6$)$ afirmaram ter sido, ou ser, portadores de doenças sexualmente transmissíveis (219 do sexo masculino e 2 do sexo feminino).

Para fins de análise, foram excluídas as mulheres $(n=5)$. Assim, dentre as variáveis investigadas, o relato de DST mostrou-se estatisticamente associado à idade $>30$ anos, escolaridade $\leq 8$ anos, tempo de profissão $>10$ anos, antecedente prisional, relações sexuais desprotegidas com parceira fixa e relações sexuais com profissional do sexo $(P<0,05)$. Essas variáveis, bem como o uso de "rebite" e a permanência fora do lar por mais de 15 dias, que se mostraram marginalmente associadas ao relato de DST, foram incluídas em um modelo hierárquico de regressão logística. Após controle das variáveis confundidoras, idade entre 41 e 50 anos (OR ajustado: 4,2; IC95\%: 1,9 a 8,7) e acima de 50 anos (OR ajustado: 5,7; IC95\%: 2,4 a 13,7), uso de "rebite" (OR ajustado: 1,7; IC95\%: 1,1 a 2,6), antecedente prisional (OR ajustado: 2,2; IC95\%: 1,2 a 4,2 ) e relações sexuais com profissionais do sexo (OR ajustado: 1,9; IC95\%: 1,3 a 2,8) mantiveram-se independentemente associadas ao relato de DST. Ainda, não usar preservativo com parceira fixa (OR ajustado: 1,7; IC95\%: 1,0 a 2,9) e faixa etária de 31 a 40 anos (OR ajustado: 2,0; IC95\%: 1,0 a 3,9) mostraram uma tendência à associação com DST (tabela 2).

Outras variáveis, como múltiplos parceiros sexuais, uso inconsistente de preservativo com parceira ocasional, não ter usado preservativo na última
TABELA 1. Características sociodemográficas de 641 caminhoneiros de rota longa que trafegavam na rodovia federal BR-153, Brasil, 2005 a 2006

\begin{tabular}{|c|c|c|}
\hline Característica & No. & $\%$ \\
\hline \multicolumn{3}{|l|}{ Sexo } \\
\hline Feminino & 5 & 0,8 \\
\hline Masculino & 636 & 99,2 \\
\hline \multicolumn{3}{|l|}{ Idade (anos) } \\
\hline$\leq 30$ & 122 & 19,0 \\
\hline 31 a 40 & 211 & 32,9 \\
\hline 41 a 50 & 192 & 30,0 \\
\hline$>50$ & 116 & 18,1 \\
\hline \multicolumn{3}{|l|}{ Estado civil } \\
\hline Casado & 498 & 77,7 \\
\hline Viúvo/separado & 44 & 6,9 \\
\hline Solteiro & 99 & 15,4 \\
\hline \multicolumn{3}{|c|}{$\begin{array}{l}\text { Renda familiar } \\
\text { (salários mínimos) }\end{array}$} \\
\hline$\leq 3$ & 183 & 28,7 \\
\hline 4 a 6 & 309 & 48,4 \\
\hline$>6$ & 146 & 22,9 \\
\hline \multicolumn{3}{|c|}{ Naturalidade (região) } \\
\hline Sudeste & 263 & 41,2 \\
\hline Sul & 258 & 40,2 \\
\hline Centro-Oeste & 49 & 7,6 \\
\hline Nordeste & 59 & 9,2 \\
\hline Norte & 12 & 1,9 \\
\hline \multicolumn{3}{|l|}{ Escolaridade (anos) } \\
\hline$>8$ & 165 & 25,7 \\
\hline 5 a 8 & 276 & 43,1 \\
\hline$\leq 4$ & 200 & 31,2 \\
\hline
\end{tabular}

a Sem informação para 3 participantes.

relação sexual, uso de álcool ou drogas ilícitas durante as relações sexuais e relações sexuais com parceiro do mesmo sexo não foram associadas estatisticamente ao relato de DST $(P>0,05)$.

\section{DISCUSSÃO}

Para o controle e a prevenção das DST, é preciso conhecer os padrões comportamentais e sociais envolvidos, identificar as populações-alvo para intervenções e elaborar estratégias de prevenção eficazes. No Brasil, ainda são poucos os estudos sobre a população de caminhoneiros $(6,11,14,15)$. Este estudo apresenta a primeira investigação em caminhoneiros que trafegam na BR-153, uma das principais rodovias federais, que atravessa o País de sul a norte. 
O Brasil possui uma frota de aproximadamente 1,8 milhão de caminhões e uma população de mais de 700000 caminhoneiros, composta quase que exclusivamente por homens, que viajam, em média, $175000 \mathrm{~km}$ por ano $(16,17)$. Neste estudo, como em outros, essa população foi composta por adultos sexualmente ativos, com escolaridade e renda familiar baixa $(8,11,18-20)$. Portanto, esses indivíduos, que possuem grande mobilidade geográfica, podem servir como disseminadores de doenças infecciosas, principalmente daquelas transmitidas sexualmente.

Utilizou-se, para definição de DST, o auto-relato de corrimento uretral ou úlcera genital associados ou não a his- tória de diagnóstico médico laboratorial de DST. Os primeiros critérios são objetivos e caracterizam os principais sintomas utilizados na abordagem sindrômica de DST em homens (21). Ainda, estudos têm mostrado "relato de DST" como preditor de comportamentos de risco (2), principalmente em homens $(3,22)$, sendo, portanto, uma ferramenta útil para identificar as populações-alvo para programas de prevenção.

A maioria dos estudos sobre as DST em caminhoneiros se origina de países asiáticos e africanos. No sul da Î́ndia, em uma clínica de DST, localizada à margem de uma rodovia, Manjunath et al. (7) encontraram história de úl- cera genital e uretrite (nos últimos 5 anos) em $31,9 \%$ e $26,2 \%$ dos 263 motoristas de caminhão estudados, respectivamente. Nesses indivíduos, a prevalência de positividade ao teste VDRL e anti-HIV foi de $13,3 \%$ e $15,9 \%$. Já Gawande et al. (10) relataram um índice de sífilis de 21,9\% em 670 indivíduos que trafegavam na região central daquele país. Ainda, dos 67 caminhoneiros que referiram corrimento uretral, 45 (67\%) eram portadores de N. gonorrhoeae. Em Bangladesh, Gibney et al. (4) encontraram uma prevalência global para infecção por C. trachomatis, N. gonorrhoeae e T. pallidum de 7,8\% em 384 caminhoneiros, enquanto na China, Chen et al. (9) de-

TABELA 2. Análise multivariada dos comportamentos de risco associados ao relato de doenças sexualmente transmissíveis em caminhoneiros brasileiros que trafegavam na rodovia federal BR-153, Brasil, 2005 a 2006

\begin{tabular}{|c|c|c|c|c|c|c|c|}
\hline Fatores de risco & Sim & $\begin{array}{l}\text { Relato } \\
\text { de DST }\end{array}$ & $\%$ & $\begin{array}{l}\text { OR }{ }^{\mathrm{a}} \text { não ajustado } \\
\text { (IC95\%) }\end{array}$ & $P$ & $\begin{array}{l}\text { ORa ajustado } \\
\text { (IC95\% })^{b}\end{array}$ & $P$ \\
\hline \multicolumn{8}{|l|}{ Idade (anos) } \\
\hline 31 a 40 & 205 & 62 & 30,2 & $2,3(1,3$ a 4,4$)$ & 0,00 & $2,0(1,0$ a 3,9$)$ & 0,05 \\
\hline 41 a 50 & 185 & 83 & 44,9 & $4,4(2,4$ a 8,2$)$ & 0,00 & $4,2(1,9$ a 8,7$)$ & 0,00 \\
\hline$>50$ & 110 & 56 & 50,9 & $5,6(2,9$ a 11,0$)$ & 0,00 & $5,7(2,4$ a 13,7$)$ & 0,00 \\
\hline \multicolumn{8}{|l|}{ Escolaridade (anos) ${ }^{c}$} \\
\hline$\leq 4$ & 159 & 67 & 42,1 & $2,6(1,6$ a 4,1$)$ & 0,00 & $0,9(0,6$ a 1,6$)$ & 0,86 \\
\hline \multicolumn{8}{|l|}{ Tempo de profissão (anos) ${ }^{c}$} \\
\hline$<10$ & 225 & 52 & 23,1 & 1,0 & & 1,0 & \\
\hline 11 a 20 & 197 & 99 & 37,6 & $2,0(1,3$ a 3,1$)$ & 0,00 & $1,3(0,8$ a 2,2$)$ & 0,29 \\
\hline$>20$ & 192 & 67 & 47,9 & $3,0(2,0$ a 4,8$)$ & 0,00 & $1,3(0,7$ a 2,4$)$ & 0,36 \\
\hline \multicolumn{8}{|c|}{ Período fora de casa (dias) ${ }^{d}$} \\
\hline \multicolumn{8}{|l|}{ Antecedente prisional $\left.\right|^{d}$} \\
\hline Não & 558 & 188 & 33,7 & 1,0 & & 1,0 & \\
\hline Sim & 55 & 31 & 56,4 & $2,5(1,4-4,4)$ & 0,00 & $2,2(1,2$ a 4,2$)$ & 0,01 \\
\hline \multicolumn{8}{|c|}{ Uso de preservativo com parceira fixa ${ }^{e}$} \\
\hline Sempre & 90 & 24 & 26,7 & 1,0 & & 1,0 & \\
\hline Ocasionalmente & 106 & 31 & 29,2 & $1,1(0,6$ a 2,2$)$ & 0,80 & $1,6(0,8$ a 3,2$)$ & 0,16 \\
\hline Nunca & 406 & 160 & 39,4 & $1,8(1,0$ a 3,1$)$ & 0,03 & $1,7(1,0$ a 2,9$)$ & 0,05 \\
\hline \multicolumn{8}{|c|}{ Contato sexual com profissionais do sexo } \\
\hline Não & 282 & 77 & 27,3 & 1,0 & & 1,0 & \\
\hline $\operatorname{Sim}$ & 333 & 142 & 42,6 & $2,0(1,4$ a 2,8$)$ & 0,00 & $1,9(1,3$ a 2,8$)$ & 0,00 \\
\hline
\end{tabular}

a OR = razão de chances (odds ratio).

${ }^{\mathrm{b}}$ Ajustado por idade, tempo de profissão, tempo fora de casa, uso de anfetamina, antecedente prisional, uso de preservativo com parceira fixa e contato sexual com profissionais do sexo.

c Sem informação para um participante.

d Sem informação para dois participantes.

e Sem informação para 13 participantes. 
tectaram essas infecções em $17,4 \%$ dos 550 indivíduos avaliados.

Nesta investigação, 35,6\% (IC95\%: 31,9 a 39,6) dos caminhoneiros referiram doenças sexualmente transmissíveis no presente ou no passado. Esse índice foi menor do que o encontrado por Lacerda et al. (11) em 300 caminhoneiros do Porto de Santos, no Estado de São Paulo (47\%; IC95\%: 41,6 a 53,1$)$; por outro lado, foi 5 vezes maior (7,1\%; IC95\%: 5,7 a 8,5) do que o encontrado por Carret et al. (3) em um estudo de base populacional realizado na área urbana de Pelotas (Estado do Rio Grande do Sul), ratificando, portanto, a grande vulnerabilidade dos caminhoneiros para essas infecções.

Verificou-se uma proporção maior de relato de DST em caminhoneiros mais velhos, variando de $15,7 \%$ em indivíduos com idade igual ou inferior a 30 anos a 50,9\% (OR ajustado: 5,7; IC95\%: 2,4 a 13,7) naqueles com mais de 50 anos. Chen et al. (9), utilizando métodos laboratoriais, também encontraram um aumento de doenças sexualmente transmissíveis diretamente proporcional à idade, sugerindo, nessa população, um risco contínuo para a aquisição dessas doenças.

Os caminhoneiros de rota longa permanecem fora do lar, muitas vezes, por várias semanas, o que favorece a procura por parceiras sexuais ocasionais, incluindo profissionais do sexo (18). Realmente, no presente estudo, independentemente da idade, verificou-se uma freqüência maior de visitas às profissionais do sexo em caminhoneiros que permaneciam fora do lar por mais de 15 dias quando comparados aos que permaneciam afastados por períodos menores (58,3 vs. $48,3 \%$ ) (dados não apresentados).

A probabilidade de exposição a uma pessoa com DST é uma função do número de novos parceiros por unidade de tempo e do número de parceiros infectados, sendo que o último é determinado pela prevalência da infecção dentro da respectiva comunidade (2). Neste estudo, a variável história de DST foi independentemente associada a antecedentes prisionais e a visitas a profissionais do sexo, grupos que apresentam índices elevados de prevalência para as $\operatorname{DST}(23,24)$.

Do total de caminhoneiros investigados, $333(54,1 \%)$ referiram contato sexual com profissionais do sexo; desses, $42,6 \%$ relataram DST. A chance de esse grupo apresentar DST foi quase o dobro em relação aos que não referiram tal comportamento. Outros autores também relatam índices elevados. $\mathrm{Na}$ África do Sul, Ramjee et al. (20) identificaram história recente de DST em $66 \%$ dos caminhoneiros que visitavam profissionais do sexo em seis postos de parada de caminhões. Em Bangladesh, Gibney et al. (18) verificaram, em caminhoneiros que tiveram três ou mais episódios de DST num intervalo de 2 anos, uma chance 5,6 (IC95\%: 1,5 a 19,9) vezes maior de relacionamento sexual com profissionais do sexo quando comparados aos que não tiveram DST.

Muitos caminhoneiros referiram o uso de álcool, inclusive durante as relações sexuais. Ainda, 13 e 30\%, respectivamente, relataram o uso de drogas ilícitas e anfetaminas, sendo que a última foi independentemente associada ao relato de DST. Existem na literatura outros relatos sobre caminhoneiros que mostram comportamentos que $(8,25)$, além de favorecer práticas sexuais de risco, podem causar acidentes nas estradas (26).

Neste estudo, assim como em outros $(5,7,9,15,18)$, observou-se uma baixa freqüência de uso de preservativos, principalmente com parceira fixa. Somente $90(14,6 \%)$ caminhoneiros afirmaram que utilizavam preservativos de forma rotineira durante as relações sexuais com parceiras fixas. Ainda, o uso consistente de preservativos com parceira ocasional, e o seu uso na última relação sexual, só foram referidos por aproximadamente metade dos caminhoneiros. Jackson et al. (5) relataram também uma baixa freqüência de uso de preservativo, principalmente com parceiros fixos, em caminhoneiros de Mombasa, África Oriental, mesmo após a participação desses em um programa de intervenção comportamental, que incluía atividades educativas de redução de risco em saúde e distribuição gratuita de preservativos. Segundo esses autores, o senso de proteção em homens, aliado à dependência financeira da parceira fixa, pode diminuir o poder de negociação da mulher em relação ao uso do preservativo.

Por fim, essa população, que apresenta baixa freqüência de uso de preservativos e elevada proporção de relato de DST e de relações sexuais com profissionais do sexo, pode funcionar como ponte entre grupos de prevalência elevada para as DST e a população em geral, disseminando essas infecções em grandes áreas geográficas em um curto espaço de tempo.

Apesar das limitações do auto-relato para avaliar as DST, o uso dessa estratégia revelou, mesmo que de forma aproximada, a prevalência de DST nos caminhoneiros. Acreditamos ainda que, mais do que superestimados, os resultados do presente estudo podem estar subestimados, uma vez que, entre outras possibilidades, alguns caminhoneiros podem ter deliberadamente negado essa experiência, enquanto outros podem ter sido ou ser portadores assintomáticos, desconhecendo, portanto, o seu estado de portador. Com essa convicção realizamos, após o término da coleta de dados, nas dependências do posto de combustível utilizado no desenvolvimento do estudo, várias atividades educativas de prevenção e promoção da saúde para os caminhoneiros e trabalhadores do local.

Em suma, os resultados deste estudo evidenciam a elevada vulnerabilidade dos caminhoneiros que trafegam na BR-153 para as DST, e poderão auxiliar os profissionais de saúde na construção de estratégias de prevenção e controle dessas doenças junto a uma categoria profissional que fica, em função das suas condições de trabalho, à margem dos serviços públicos de saúde.

Agradecimentos. Este estudo teve apoio do Conselho Nacional de Desenvolvimento Científico e Tecnológico (CNPq) (processo $\mathrm{n}^{\circ}$ 473938/2004-9) e da Fundação de Apoio à Pesquisa (FUNAPE). 


\section{REFERÊNCIAS}

1. World Health Organization. Global prevalence and incidence of selected curable sexually transmitted infections-overview and estimates. Genebra: WHO; 2001.

2. Tanfer K, Cubbins LA, Billy JO. Gender, race, class and self-reported sexually transmitted disease incidence. Fam Plann Perspect. 1995;27(5):196-202.

3. Carret MLV, Fassa AG, Silveira DS, Bertoldi AD, Hallal PC. Sintomas de doenças sexualmente transmissíveis em adultos: prevalência e fatores de risco. Rev Saude Publica. 2004; 38(1):76-84.

4. Gibney L, Saquib N, Macaluso M, Hasan KN, Aziz MM, Khan AY, et al. STD in Bangladesh's trucking industry: prevalence and risk factors. Sex Transm Infect. 2002;78(1):31-6.

5. Jackson DJ, Rakwar JP, Richardson BA, Mandaliya K, Chohan BH, Bwayo JJ, et al. Decreased incidence of sexually transmitted diseases among trucking company workers in Kenya: results of a behavioural risk-reduction programme. AIDS. 1997;11(7):903-9.

6. Malta M, Bastos FI, Pereira-Koller EM, Cunha MD, Marques C, Strathdee SA. A qualitative assessment of long distance truck drivers' vulnerability to HIV/AIDS in Itajaí, southern Brazil. AIDS Care. 2006;18(5):489-96.

7. Manjunath JV, Thappa DM, Jaisankar TJ. Sexually transmitted diseases and sexual lifestyles of long-distance truck drivers: A clinico-epidemiologic study in south India. Int J STD AIDS. 2002;13(9):612-7.

8. Nascimento EC, Nascimento E, Silva JP. Uso de álcool e anfetaminas entre caminhoneiros de estrada. Rev Saude Publica. 2007;41(2): 290-3.

9. Chen XS, Yin YP, Gong XD, Liang GJ, Zhang WY, Poumerol G, et al. Prevalence of sexually transmitted infections among long-distance truck drivers in Tongling, China. Int J STD AIDS. 2006;17(5):304-8.

10. Gawande AV, Vasudeo ND, Zodpey SP, Khandait DW. Sexually transmitted infec- tions in long distance truck drivers. J Commun Dis. 2000;32(3):212-5.

11. Lacerda R, Gravato N, McFarland W, Rutherford G, Iskrant K, Stall R, et al. Truck drivers in Brazil: prevalence of HIV and other sexually transmitted diseases, risk behavior and potential for spread of infection. AIDS. 1997;11 Suppl 1:S15-9.

12. Brasil, Ministério da Saúde. Guia de vigilância epidemiológica. $6^{\mathrm{a}}$ ed. Brasília: Ministério da Saúde; 2005. Disponível em: http:// bvsms.saude.gov.br/bvs/publicacoes/Guia Vig_Epid_novo2.pdf. Acessado em abril de 2008.

13. Victora CG, Huttly SR, Fuchs SC, Olinto MT The role of conceptual frameworks in epidemiological analysis: a hierarchical approach. Int J Epidemiol. 1997;26(1):224-7.

14. Berra JAP, Bacetti LB, Alves KJF, Fiório VLP. Soroprevalência de HIV em caminhoneiros usuários da Rodovia Anhanguera, SP 330, Brasil. Rev Inst Adolfo Lutz. 2003;62(3): 171-6

15. Villarinho L, Bezerra I, Lacerda R, Latorre MRDO, Paiva V, Stall R, et al. Caminhoneiros de rota curta e sua vulnerabilidade ao HIV, Santos, SP. Rev Saude Publica. 2002;36(4 Supl):61-7.

16. Confederação Nacional do Transporte. O perfil sócio-econômico e as aspirações dos caminhoneiros no País. 1999. Disponível em: http://www.cnt.org.br/ . (Informações. Pesquisas e boletins. Pesquisas. Perfil socioeconômico dos trabalhadores autônomos). Acessado em abril de 2008.

17. Confederação Nacional do Transporte. Boletim estatístico 2007. Disponível em: http:// www. cnt.org.br/. (Informações. Pesquisas e boletins. Boletim estatístico). Acessado em abril de 2008 .

18. Gibney L, Saquib N, Metzger J. Behavioral risk factors for STD/HIV transmission in Bangladesh's trucking industry. Soc Sci Med. 2003;56(7):1411-24
19. Medeiros MGF, Barbosa Jr. A, Cerqueira PTJ Caminhoneiros e o conhecimento sobre os meios de transmissão do HIV. Bol Epidemiol AIDS. 2001. Disponível em: http://www. aids.gov.br/data/documents/storedDocuments/\%7BB8EF5DAF-23AE-4891-AD361903553A3174\%7D/\%7B9E984A91-D50B4421-9C0D-7A1BC0D9BCB2\%7D/Boletim Epidemiol\%F3gico_2001_II_Aids.pdf. Acessado em abril de 2008.

20. Ramjee G, Gouws E. Prevalence of HIV truck drivers visiting sex workers in KwaZuluNatal, South Africa. Sex Transm Dis. 2002; 29(1):44-9.

21. Brasil, Ministério da Saúde. Manual de bolso das doenças sexualmente transmissíveis. 2a ed. Brasília: Ministério da Saúde; 2006.

22. Warszawski J, Meyer L. Gender difference in persistent at-risk sexual behavior after a diagnosed sexually transmitted disease. ACSFInvestigators. Sex Transm Dis. 1998;25(8): $437-42$.

23. Miranda AE, Vargas PM, St Louis ME, Viana MC. Sexually transmitted diseases among female prisoners in Brazil: prevalence and risk factors. Sex Transm Dis. 2000;27(9):491-5.

24. Ndoye I, Mboup S, De Schryver A, Van Dyck E, Moran J, Samb ND, et al. Diagnosis of sexually transmitted infections in female prostitutes in Dakar, Senegal. Sex Transm Infect. 1998;74 Suppl 1:S112-7.

25. Souza JC, Paiva T, Reimão R. Sleep habits, sleepiness and accidents among truck drivers. Arq Neuropsiquiatr. 2005;63(4):925-30.

26. Crouch DJ, Birky MM, Gust SW, Rollins DE, Walsh JM, Moulden JV, et al. The prevalence of drugs and alcohol in fatally injured truck drivers. J Forensic Sci. 1993;38(6):1342-53.

Manuscrito recebido em 14 de agosto de 2007. Aceito em versão revisada em 21 de janeiro de 2008.
ABSTRACT

Risk behaviors for sexually transmitted diseases among truck drivers in Brazil
Objective. To evaluate the frequency of sexually transmitted diseases (STD) and associated risk factors based on self-reporting by long-haul truck drivers in Brazil.

Method. From October 2005-October 2006, 641 long-haul truck drivers that travel federal highway BR-153, traversing the country from south to north, were interviewed. A structured interview was used to collect sociodemographic data. The truck drivers also completed a self-administered questionnaire on risk behaviors and STD history. The data were adjusted and analyzed using logistic regression.

Results. Of 641 drivers interviewed, 620 (96.7\%) provided answers on STD history. Of these, $35.6 \%(95 \%$ confidence interval $(\mathrm{CI})=31.9-39.6)$ reported past or current STD. Being 30 years of age or older was statistically associated with having a history of STD. In addition, truck drivers who reported using amphetamines (odds ratio $(\mathrm{OR})=1.7$; $95 \% \mathrm{CI}=1.1-2.6)$, having been incarcerated $(\mathrm{OR}=2.2 ; 95 \% \mathrm{CI}=1.2-4.2)$, and sexual relations with sex professionals $(\mathrm{OR}=1.9 ; 95 \% \mathrm{CI}=1.3-2.8)$ had increased odds for having a history of STD.

Conclusion. These results confirm that Brazilian truck drivers are highly vulnerable to STD and show the importance of prevention programs targeting this specific group, one that is constantly on the move and may efficiently disseminate STD.

Truck drivers, sexually transmitted diseases, risk factors, Brazil. 